\title{
Enhancing spectral contrast in organic red-light photodetectors based on a light-absorbing and exciton-blocking layered system
}

\author{
Yusuke Higashi, ${ }^{1}$ Kyu-Sik Kim, ${ }^{2}$ Hyeon-Gu Jeon, ${ }^{1}$ and Musubu Ichikawa ${ }^{1, a)}$ \\ ${ }^{1}$ Functional Polymer Science Course, Faculty of Textile Science and Technology, Shinshu University, 3-15-1 \\ Tokida, Ueda City, Nagano 386-8567, Japan \\ ${ }^{2}$ Samsung Advanced Institute of Technology, Samsung Electronics, Mt. 14-1, Nongseo-Dong, Giheung-Gu, \\ Yongin-Si 446-712, Republic of Korea
}

(Received 6 May 2010; accepted 20 June 2010; published online 3 August 2010)

\begin{abstract}
We demonstrated a highly sensitive red-light photodetector based on a mixed copper phthalocyanine $(\mathrm{CuPc})$ and fullerene $\mathrm{C}_{60}$ photoactive layer, similar to a so-called bulk heterojunction structure usually used in the field of organic photovoltaics. We incorporated an additional set of organic layers that was composed of two organic p-type semiconductors to reduce the blue-light sensitivities of CuPc- and $\mathrm{C}_{60}$-based organic photodetectors. We used $\alpha, \omega$-diphenyl sexi-thiophene (P6T) and $\alpha, \omega$-bis(biphenyl-4-yl)ter-thiophene (BP3T), which are thiophene-based materials and usually have good hole-transporting properties. A thick $(>100 \mathrm{~nm})$ P6T layer absorbed blue light, preventing it from reaching the photoactive layer, and a thin $(\sim 20 \mathrm{~nm})$ BP3T layer whose band gap was larger than that of P6T blocked excitation energy transfer from P6T to CuPc. Thus, we successfully demonstrated a red-light photodetector with high peak sensitivity and whose current-voltage characteristics did not worsen. The optimal device showed a peak incident photon-current conversion efficiency of $51.7 \%$ at $620 \mathrm{~nm}$ and a specific detectivity of $4.0 \times 10^{11} \mathrm{~cm} \mathrm{~Hz}^{1 / 2} / \mathrm{W}$.

(c) 2010 American Institute of Physics. [doi:10.1063/1.3466766]
\end{abstract}

\section{INTRODUCTION}

Our information-age society has a seemingly insatiable need for electronic devices, which are increasingly smaller and cheaper while simultaneously offering more functionality. This need has led to intensive searches for alternative semiconducting materials. Organic semiconductors appear to be very promising for many applications compared to their inorganic counterparts. as they offer numerous desirable properties and technological advantages, i.e., mechanical flexibility, produced devices and systems with large area, simplicity of processing, and, consequently, low cost. ${ }^{1-3}$ Much recent attention is being paid to photovoltaic (PV) cells, ${ }^{4-6}$ and more recently to organic photodetectors (OPDs).$^{7-12}$ In contrast to OPDs, solar cells require a high conversion efficiency over the entire solar spectrum, which reaches significantly into the near-infrared, whereas organic semiconductors with narrow band gaps are still being developed intensively. ${ }^{13,14}$ However, photodectors require light sensitivity of high and sensitive spectral regions of them depend on their applications. As organic materials such as dyes and pigments are some of the most useful color materials, we believe that color-sensitive photodectors based on organic materials are among applications with the most potential.

Moreover, organic color-sensitive photosensors are probably able to provide another advantage to full-color image sensors. Recent high resolution full-color image sensors that are used for digital still camera, camcorders, and so on are mostly composed of an Si-based photodetector array and a color filter array where a lot of small color filters for each

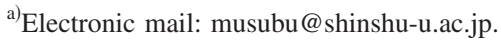

primary color of light are placed side by side. The color filters selectively transmit a primary color of light from target lights, and in other words, the color filter arrays waste two in three of the lights for full-color imaging. Consequently, it is concerned that this will lead to limiting to obtain higher resolution image sensors with sufficient light sensitivity. On the other hand, organic material-based photodetectors will be able to provide color selective sensitivity because some of organic materials such as phthalocyanines, perylenetetracarboxylic diimides, azo derivatives, and so on show brilliant colors. These color selectivity can provide another structure to fabricate full color image sensors; a layer stack type resembling color photographic films and papers as shown in Fig. 1(a). ${ }^{15-17}$ This might change full-color digital image sensors smaller, more lightweight, and higher resolution. ${ }^{16}$

In the case of layer-stack type image sensors, we need to consider the order of the subcells for each primary color of light. From the viewpoint of physical chemistry of organic materials, red-light sensitive subcells (R-cells), of course, mainly absorb red light but also slightly absorb blue and green lights fundamentally because red-light-absorbing molecules must have other electronic transitions due to higher excite states. In the case of almost all organic color materials these nonpreferable electronic transitions are weak but not zero, and this gives a nonpreferable sensitivity for R-cells as shown in Fig. 1(b). Consequently, when we consider this fact for determining the order of the subcells it will be the best to place a R-cell at the bottom and blue light sensitive subcell (B-cell) at the top as shown in Fig. 1(a). This is because the B-cell absorbs blue light in no small measure and reduce the intensity of nonpreferable blue light reaching into the under 
(a)

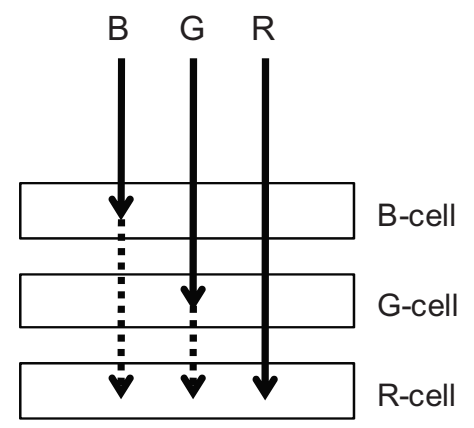

(b)

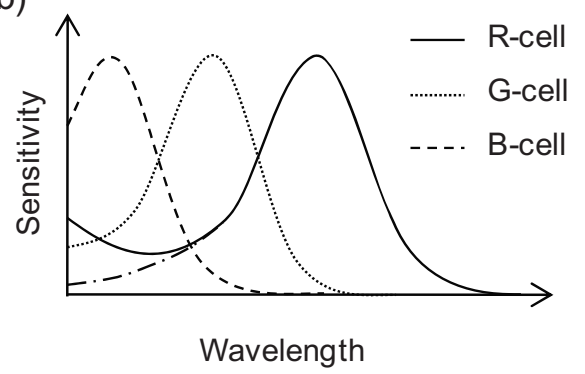

FIG. 1. (a) Schematic illustration of device structure and light absorptions by each cell in a vertical stack full-color image sensor. (b) Schematic sensitivity spectra for each cell. Dashed-dotted line in panel (b) schematically depicts a compensated sensitivity curve of an R-cell.

cells. The green light sensitive sub cell (G-cell) also has the same effect for green light. However, at present it is very difficult to absorb light completely by upper sub cells as shown by the dotted line arrows in Fig. 1(a) because of the limit to organic PV; short exciton diffusion length and low charge carrier mobility in organic semiconducting materials require thin photoactive organic layers. ${ }^{18,19}$ Consequently, development of spectral sensitivity compensation technologies that can be built-in in subcells is very important. Figure 1(b) also schematically depicts the compensation by a dashed-dotted line.

High sensitivities are very important for image sensors, and the organic $\mathrm{p} / \mathrm{n}$ junction based on $\mathrm{p}$ - and $\mathrm{n}$-types organic semiconducting materials is very important for getting high photon-to-electron conversion quantum efficiencies. In this context, fullerene $\mathrm{C}_{60}$ must be undoubtedly the most useful n-type organic semiconductor in this field, and indeed, almost all organic PVs with high conversion efficiencies are made of fullerene $\mathrm{C}_{60}$ derivatives despite their weak light absorption in the visible and near-infrared light regions. It is widely believed that a high quantum efficiency of photoinduced charge separation in pairs of fullerenes and p-type organic semiconductors such as phthalocyanines, pentacene, and thiophenes is one of the keys for high power conversion efficiency. While fullerenes show weak absorption in the visible light region because of their highly symmetrical structure, they show strong absorption in the blue and ultraviolet regions. Considering subcells for each primary color of light, fullerenes must be quite useful in the case of B-cells, but spectral compensation would be required in the case of Gand R-cells. Consequently, in this paper we will demonstrate a spectral sensitivity compensation for red-light detection for

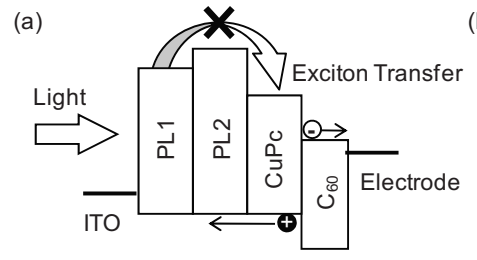

(b)

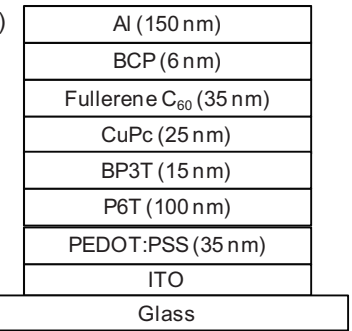

(c)
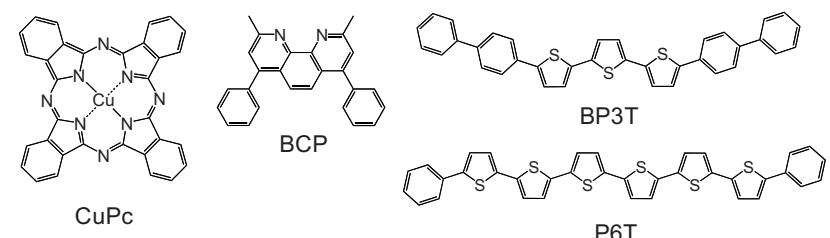

P6T

FIG. 2. (a) Conceptual energy diagram of red-light-only sensitive organic photodiode based on $\mathrm{CuPc}$ and $\mathrm{C}_{60}$, (b) sectional device structure, and (c) structures of chemicals and their abbreviations used.

the phthalocyanine- and $\mathrm{C}_{60}$-based OPD by using a lightabsorbing and exciton-blocking layered system.

\section{EXPERIMENTAL}

\section{A. Device structure}

Figure 2 shows the schematic energy diagram of the device prepared in this study. Light is incident on the indium tin oxide (ITO) layer, and a copper phthalocyanine (CuPc) and $\mathrm{C}_{60}$ planar or bulk (mixed) heterojunction converts photons to electrons, where $\mathrm{CuPc}$ is a good red-light absorber. We placed two additional p-type semiconducting layers (PL1 and PL2) in front of the photoactive heterojunctions. As is known, a combination of $\mathrm{CuPc}$ and $\mathrm{C}_{60}$ is one of the best combinations for photon-electron conversions, ${ }^{20}$ and this combination, leads to high incident photon-current conversion efficiency (IPCE) in wavelengths of both blue and red light. To reduce IPCE for blue light due to $\mathrm{C}_{60}$, we thus used the two additional p-type semiconducting layers. As shown in Fig. 3, a thiophene derivative P6T as PL1 absorbs blue light and also green light to some degree; this means that using $\mathrm{CuPc}$ and $\mathrm{C}_{60}$, the P6T layer can reduce the intensities of blue and green light that arrive at the heterojunction. The light absorption by P6T must give rise to P6T excitons that potentially move into the CuPc layer based on excitation energy transfer because of P6T has a wider band gap than $\mathrm{CuPc}$, as shown in the inset of Fig. 3. In fact, the transfer of

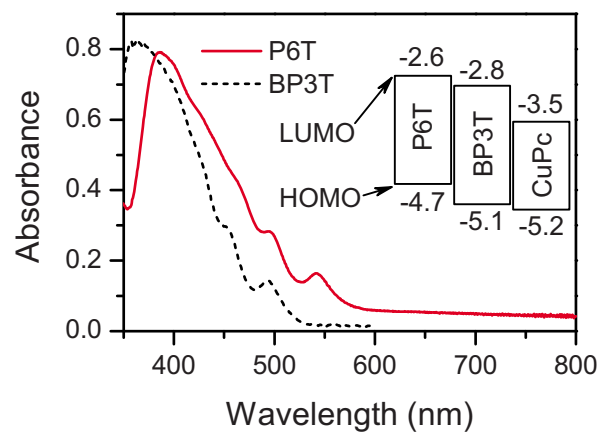

FIG. 3. (Color online) Absorption spectra of P6T and BP3T thin films. The inset shows the HOMO and LUMO levels of P6T, BP3T, and CuPc. 
the exciton led to increasing IPCE at the blue-light region where P6T absorbed light if the thickness of P6T was 40 $\mathrm{nm} .{ }^{21}$ In contrast, by placing BP3T, another thiophene derivative, as PL2 between P6T and the photoactive layers, as shown in Fig. 2, the nonpreferable excitation transfer probably should be stopped because BP3T has a wider band gap than P6T. Note that the light potentially absorbed by BP3T nearly disappears after passing through the P6T layer, as shown in Fig. 3.

\section{B. Device fabrication and measurements}

Figure 2 also shows the chemical structures of the materials used. As the additional p-type semiconducting material layers should possess a good hole-transporting ability (thiophene/phenylene), co-oligomers such as BP3T and P6T have already been studied and reported. ${ }^{22-25}$ The following chemicals, BP3T and P6T (Sumitomo Seika Chemicals), and bathocuproine (BCP), Tokyo Chemical Industry (TCI) were purified by temperature gradient train sublimation with $\mathrm{Ar}$ gas flow before use. We used $\mathrm{C}_{60}$, purchased from Frontier carbon (nanom purple SUH grade), and $\mathrm{CuPc}$ (for the organic light-emitting device) donated by Nippon Steel Chemicals, without further purification. We prepared the devices shown in Fig. 2(b) by depositing several materials sequentially onto commercially available ITO-coated glass substrates that had sheet resistances of $15 \Omega /$ sq. First, the substrates were washed with detergent water, pure water, and 2-propanol under ultrasonification and treated with $\mathrm{O}_{2}$ plasma at a power of $50 \mathrm{~W}$ for $5 \mathrm{~min}$. We prepared a poly(3,4-ethylenedioxythiophene) poly(styrenesulfonate) (PEDOT:PSS) layer on the substrates by spin-coating a commercially available formula (H.C. Starck, Clevios P Al 4083). We deposited several organic layers by thermal evaporation under vacuum $\left(2.0 \times 10^{-4} \mathrm{~Pa}\right)$ at a rate of $1 \AA / \mathrm{s}$. Finally, the aluminum electrode was prepared on the top of the organic layer stack by thermal evaporation in the same vacuum at a rate of $10 \AA / \mathrm{s}$. Active areas of the cells were about $6 \mathrm{~mm}^{2}$, and we confirmed the areas one by one using a microscope. We transferred the produced devices into a highly inert glove box $\left(\mathrm{O}_{2}\right.$ concentration $5 \mathrm{ppm}$; dew point $<-50{ }^{\circ} \mathrm{C}$ ) with no exposure to ambient conditions and then mounted them in a small chamber with a quartz window in the glove box. A source meter (Keithley, 2410) measured their current density versus voltage (J-V) curves both with and without $100 \mathrm{~mW} / \mathrm{cm}^{2}$ artificial solar light irradiation of air mass 1.5 global (AM1.5G) from a Newport Oriel solar simulator (Model 91160) with an AM1.5G filter (Newport Oriel, 81088) and a light intensity stabilizer (Newport Oriel, Model 68945). The power density of irradiation was measured with a pyroelectric optical power meter (Ophir, P3). We recorded the IPCE spectra of the devices with a digital multimeter (Advantest, AD7461A) at zero bias or a source meter (Keithley, 2400) at negative biases under monochromatic light irradiation from a light source (Bunko Keiki, SM$25 \mathrm{~A}$ ), where the intensity of the monochromatic light for each wavelength was measured with a calibrated silicon photodiode (Hamamatsu Photonics, S1337-1010BQ).
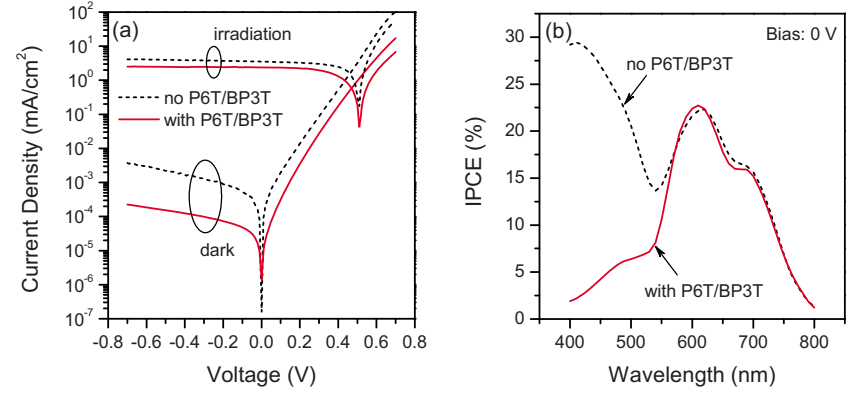

FIG. 4. (Color online) (a) J-V characteristics in dark or $100 \mathrm{~mW} / \mathrm{cm}^{2}$-AM1.5G irradiation and (b) IPCE spectra of the device with a planar heterojunction whose structure is $\mathrm{ITO} / \mathrm{P} 6 \mathrm{~T} / \mathrm{BP} 3 \mathrm{~T} / \mathrm{CuPc} / \mathrm{C}_{60} / \mathrm{BCP} / \mathrm{Al}$, shown together with those of the reference device (no P6T and BP3T layers).

\section{RESULTS AND DISCUSSION}

\section{A. Planar heterojunction devices}

Figure 4(a) shows J-V curves of two devices based on the $\mathrm{CuPc} / \mathrm{C}_{60}$ planar heterojunction with and without the two-layers set, P6T and BP3T. Both devices showed clear rectifying behavior, and the device with the two-layer set showed a smaller reverse dark current at $-0.7 \mathrm{~V}$ than the device without the set. Incorporating the additional layers makes the device thicker, and this probably reduced dark current at reverse bias. As shown in Fig. 4(a), fill factors (FFs) of both devices are, however, almost the same $(0.50$ and 0.60 for devices with and without the layers), and this result indicates that the set of layers did not make the device highly resistive. Both good hole mobilities of P6T and BP3T (Ref. 24) and well-aligned highest occupied molecular orbital (HOMO) levels of P6T, BP3T, and CuPc shown in the inset of Fig. 3 probably led to keeping low resistivity in the case of the introduced P6T and BP3T layers. In addition, open circuit voltages $\left(\mathrm{V}_{\mathrm{OC}}\right)$ of both cells are almost the same as shown in the figure. This fact can be confirmed by a general sense that $\mathrm{V}_{\mathrm{OC}}$ depends on the difference between the HOMO energy of the p-type and the lowest unoccupied molecular orbital (LUMO) energy of the n-type semiconductors, which are used to build the $\mathrm{p} / \mathrm{n}$ junction.

Figure 4(b) shows IPCE spectra of both devices. The device $\left(\mathrm{CuPc} / \mathrm{C}_{60}\right)$ showed photon-to-electron conversion at both red- and blue-light regions in which $\mathrm{CuPc}$ and $\mathrm{C}_{60}$ each independently contribute to the conversion, respectively. In contrast, the device with the P6T and BP3T layers still showed sensitivity against red light around $620 \mathrm{~nm}$, though it had much less sensitivity for blue light. The incorporation of P6T and BP3T layers led to both decreasing the conversion efficiency for blue light and keeping the sensitivity for red light. If a P6T layer was directly attached to the CuPc layer, it would have appeared that decreasing of IPCE at blue light was not very large. We will discuss the effect of BP3T, the exciton blocking layer below. While the $\mathrm{J}-\mathrm{V}$ curve of the device with P6T and BP3T layers showed smaller photocurrent than the device without the layers [Fig. 4(a)], the decrease probably results from blinding against blue light. 

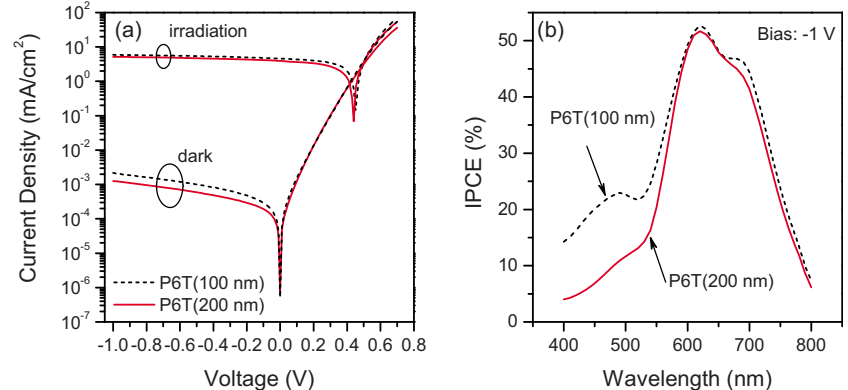

FIG. 5. (Color online) (a) J-V characteristics in dark or $100 \mathrm{~mW} / \mathrm{cm}^{2}$-AM1.5G irradiation and (b) IPCE spectra of the mixed photoactive layer devices with a 100 or 200-nm-thick P6T layer. The device structure is $\mathrm{ITO} / \mathrm{P} 6 \mathrm{~T} / \mathrm{BP} 3 \mathrm{~T} / \mathrm{CuPc} / \mathrm{CuPc}: \mathrm{C}_{60} / \mathrm{C}_{60} / \mathrm{BCP} / \mathrm{Al}$.

\section{B. Bulk heterojunction devices}

Figure 5(b) shows IPCE spectra for a bulk heterojunction device having a mixed photoactive layer composed of $\mathrm{CuPc}$ and $\mathrm{C}_{60}$. The structure of the device was ITO/ PEDOT:PSS(35 nm)/P6T(100 or $200 \mathrm{~nm}) / \mathrm{BP} 3 \mathrm{~T}(20 \mathrm{~nm}) /$ $\mathrm{CuPc}(10 \mathrm{~nm}) / \mathrm{CuPc}: \mathrm{C}_{60}(30 \mathrm{~nm}) / \mathrm{C}_{60}(20 \mathrm{~nm}) / \mathrm{BCP}(6 \mathrm{~nm}) /$ $\mathrm{Al}$, and the mixing ratio of $\mathrm{CuPc}$ and $\mathrm{C}_{60}$ was $1: 1$ by weight. Note that we treated the devices thermally after depositing the mixed layer in the glove box at $100{ }^{\circ} \mathrm{C}$, and then, thermally deposited were the other layers onto the devices in the vacuum chamber again. As shown in the figure, employing a mixed photoactive layer enhanced IPCE for both red and blue light. The enhancement on the conversion by mixing phthalocyanine and fullerene has been reported in the field of organic PV cells, ${ }^{26}$ and the mechanism of the IPCE enhancement observed in this study is probably the same as in the case of organic PV cells. Here we define spectral contrast ratio (SCR) as an IPCE ratio at 620 and $450 \mathrm{~nm}$. Although the incorporation of the mixed $\mathrm{CuPc}: \mathrm{C}_{60}$ layer caused a high peak IPCE at $620 \mathrm{~nm}$ of $52.7 \%$, it also worsens SCR. The device with a thicker P6T layer at a thickness of $200 \mathrm{~nm}$ and the same mixed $\mathrm{CuPc}: \mathrm{C}_{60}$ layer accomplished both a high peak IPCE of $51.7 \%$ at $620 \mathrm{~nm}$ and a good SCR of 7.4. The IPCE value of $51.7 \%$ at $620 \mathrm{~nm}$ translated into a responsivity of $0.26 \mathrm{~A} / \mathrm{W}$. Surprisingly, the thick P6T device showed almost comparable $\mathrm{J}-\mathrm{V}$ characteristics in dark and under the irradiation [Fig. 5(a)], and it showed lower dark current at reverse bias. FFs of both $\mathrm{J}-\mathrm{V}$ curves under the irradiation were 0.51 and 0.46 for the 100 and 200-nm-thick P6T device, and a dark current at $-1 \mathrm{~V}$ of the 200-nm-thick P6T device was $1.3 \mu \mathrm{A} / \mathrm{cm}^{2}$. Here we can conclude that we have successfully developed a new method that reduced sensitivity for blue light without degradations of other characteristics. Note that the sensitivities' tail below $550 \mathrm{~nm}$ should be of no matter because pixels of full-color image sensors for three principal lights must have a spectral overlap in each sensitive region to reproduce intermediate colors such as sky blue, yellow, and orange, etc., as shown in Fig. 1(a). Similar overlaps of spectral sensitivities are found in three types of color receptor cells in human eye. ${ }^{27}$

As already mentioned in the above paragraph, the thin BP3T layer was placed for the purpose of blocking exciton transfer from P6T to CuPc since the exciton transfer potentially occurs. This is because we have previously reported

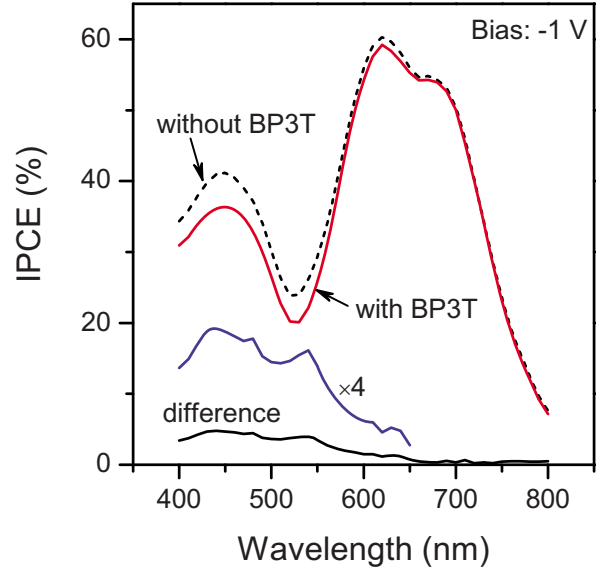

FIG. 6. (Color online) IPCE spectra of the mixed photoactive layer devices with or without BP3T layer. Thickness of P6T layer was $50 \mathrm{~nm}$.

that such interlayer excitation energy transfer can be useful for organic PV devices. ${ }^{21}$ However, it needs to clarify the effect of the thin BP3T layer experimentally. Figure 6 shows IPCE spectra for devices with or without the BP3T layer. The structure of the device with BP3T is the same as the former cell having the mixed photoactive layer expect for the thickness of its P6T layer. We changed the thickness for making the block-effect clearer from the viewpoint of the exciton diffusion length in P6T layers. We are not sure about the length at present, but we have already reported if thickness of P6T was $40 \mathrm{~nm}$, we could observe increasing IPCE due to exciton transfer from P6T to CuPc layers. Here we set the thickness to be $50 \mathrm{~nm}$. As one can see from Fig. 6, a cell without BP3T showed higher IPCEs than that with BP3T in a wavelength region below about $600 \mathrm{~nm}$. The same figure also showed the difference spectrum of both IPCE spectra and the four times vertically scaled-up one. The difference spectrum resembled the absorption spectrum of P6T thinfilm shown in Fig. 3 more than that of BP3T. If the IPCE decrease resulted from light absorption by the additionally inserted BP3T layer, the difference spectrum must be similar to the absorption spectrum of BP3T. Consequently, it was concluded that the thin BP3T layer worked as the exciton blocking layer. Note that the 50-nm-thick P6T device showed a higher IPCE at $620 \mathrm{~nm}$ than the 100 and 200-nmthick P6T devices shown in Fig. 5(b). We believe that one of the reasons for this might be a lower resistivity due to the thinner P6T layer. We measured these IPCE spectra under the same bias of $-1 \mathrm{~V}$ from the viewpoint of practical applications.

\section{Linearity and detectivity}

Figure 7 shows the irradiation monochromatic $(620 \mathrm{~nm})$ light intensity dependence of the output current response of the device with the 200-nm-thick P6T layer and the mixed photoactive layer. The device showed a good linear output characteristic for the red light, and the responsivity for the red light was $0.28 \mathrm{~A} / \mathrm{W}$ from the slope of the fitting line shown in the figure. The responsivity of $0.28 \mathrm{~A} / \mathrm{W}$ is a bit different from the other responsivity of $0.26 \mathrm{~A} / \mathrm{W}$ obtained in the above section. The difference means that the red-light 


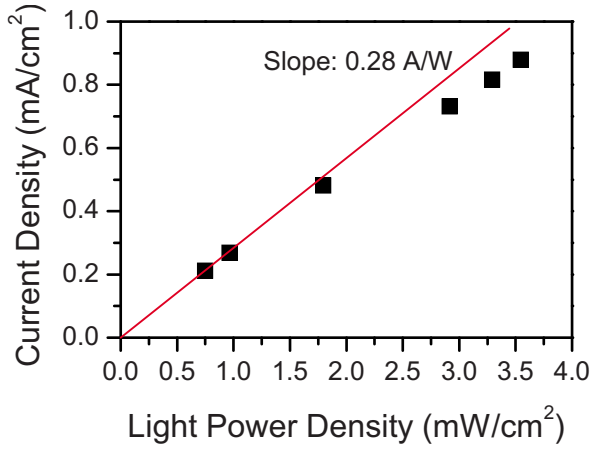

FIG. 7. (Color online) Input light (wavelength of $620 \mathrm{~nm}$ ) power density dependence of output current density of the mixed photoactive layer device with a 200-nm-thick P6T layer.

detector showed slight saturated output behavior, and an output current probably decreases about $10 \%$ at an input light intensity of $3.5 \mathrm{~mW} / \mathrm{cm}^{2}$. We are currently unsure about the origin of these changes, but we think that the decrease probably results from optical absorption of charge carriers (cations of $\mathrm{CuPc}, \mathrm{P} 6 \mathrm{~T}$, and $\mathrm{BP} 3 \mathrm{~T}$ and/or anions of $\mathrm{C}_{60}$ ) and/or bleaching of potentially red-light-absorbing $\mathrm{CuPc}$ in its ground state.

Finally, we would like to briefly discuss the detector noise of the device. The small dark current of $1.3 \mu \mathrm{A} / \mathrm{cm}^{2}$ at $-1 \mathrm{~V}$ of the device whose active area was $6.8 \mathrm{~mm}^{2}$ translated into a noise equivalent power (NEP) of 6.5 $\times 10^{-13} \mathrm{~W} / \mathrm{Hz}^{-1 / 2}$ in the case of the dark current limit. ${ }^{28}$ For comparison with detectors of different area, we introduce the specific detectivity parameter defined by

$$
D^{*}=\frac{\sqrt{A}}{\mathrm{NEP}}
$$

where $D^{*}$ is the specific detectivity and $A$ is the active area of the device. A high $D^{*}$ indicates the ability to detect lower levels of radiant power. This factor produces a figure of merit that is area-independent. We obtained a specific detectivity of $4.0 \times 10^{11} \mathrm{~cm} \mathrm{~Hz}^{1 / 2} / \mathrm{W}$ for the optimized red-light detector, which was not much worse than a well-established inorganic silicon photodiode like, for instance, a Hamamatsu S2551 with a specific detectivity of $1.5 \times 10^{13} \mathrm{~cm} \mathrm{~Hz}^{-1 / 2} / \mathrm{W}$.

\section{SUMMARY}

In summary, we demonstrated a highly sensitive redlight photodetector based on a mixed $\mathrm{CuPc}$ and $\mathrm{C}_{60}$ photoactive layer similar to a so-called bulk heterojunction structure usually used in the field of organic PVs. We incorporated an additional set of organic layers that was composed of two organic p-type semiconductors to reduce blue-light sensitivities of CuPc- and $\mathrm{C}_{60}$-based OPDs. We used P6T and BP3T, which are thiophene based and have good hole-transporting properties. A thick $(>100 \mathrm{~nm})$ P6T layer absorbed blue light, preventing blue light from reaching the photoactive layer, and a thin $(\sim 20 \mathrm{~nm})$ BP3T layer whose band gap is larger than that of P6T blocked excitation energy transfer from P6T to CuPc. Thus, we successfully demonstrated a red-light photodetector incorporating the set of P6T/BP3T layers; the photodector had high peak sensitivity and current-voltage characteristics of that did not worsen. Thus, the P6T/BP3T layers worked as a light-absorbing and exciton-blocking layered system. The optimal device showed a peak IPCE of $51.7 \%$ and a specific detectivity of 4.0 $\times 10^{11} \mathrm{~cm} \mathrm{~Hz}^{1 / 2} / \mathrm{W}$.

\section{ACKNOWLEDGMENTS}

This article is dedicated to the first principal, Chotaro Harizuka, on the occasion of 100th anniversary of Faculty of Textile Science and Technology, Shinshu University.

${ }^{1}$ G. Gustafsson, Y. Cao, G. M. Treacy, F. Klavetter, N. Colaneri, and A. J. Heeger, Nature (London) 357, 477 (1992).

${ }^{2}$ C. J. Brabec, F. Padinger, J. C. Hummelen, R. A. J. Janssen, and N. S. Sariciftci, Synth. Met. 102, 861 (1999).

${ }^{3}$ S. R. Forrest, Nature (London) 428, 911 (2004).

${ }^{4}$ J. Y. Kim, K. Lee, N. E. Coates, D. Moses, T.-Q. Nguyen, M. Dante, and A. J. Heeger, Science 317, 222 (2007).

${ }^{5}$ K. Sakai and M. Hiramoto, Mol. Cryst. Liq. Cryst. 491, 284 (2008).

${ }^{6}$ H.-Y. Chen, J. Hou, S. Zhang, Y. Liang, G. Yang, Y. Yang, Y. Wu, L. Yu, and G. Li, Nat. Photonics 3, 649 (2009).

${ }^{7}$ P. Peumans, A. Yakimov, and S. R. Forrest, J. Appl. Phys. 93, 3693 (2003).

${ }^{8}$ H. Tanaka, T. Yasuda, K. Fujita, and T. Tsutsui, Adv. Mater. (Weinheim, Ger.) 18, 2230 (2006).

${ }^{9}$ T. N. Ng, W. S. Wong, M. L. Chabinyc, S. Sambandan, and R. A. Street, Appl. Phys. Lett. 92, 213303 (2008).

${ }^{10}$ M. Ramuz, L. Bügi, C. Winnewisser, and P. Seitz, Org. Electron. 9, 369 (2008).

${ }^{11}$ H. Shimada, S. Naka, H. Okada, and H. Onnagawa, Jpn. J. Appl. Phys., Part 1 44, 3830 (2005).

${ }^{12}$ T. Someya, Y. Kato, S. Iba, Y. Noguchi, T. Sekitani, H. Kawaguchi, and T. Sakurai, IEEE Trans. Electron Devices 52, 2502 (2005).

${ }^{13}$ J. Peet, J. Y. Kim, N. E. Coates, W. L. Ma, D. Moses, A. J. Heeger, and G. C. Bazan, Nature Mater. 6, 497 (2007).

${ }^{14}$ M. M. Wienk, M. Turbiez, J. Gilot, and R. A. J. Janssen, Adv. Mater. (Weinheim, Ger.) 20, 2556 (2008).

${ }^{15}$ S. Aihara, Y. Hirano, T. Tajima, K. Tanioka, M. Abe, N. Saito, N. Kamata, and D. Terunuma, Appl. Phys. Lett. 82, 511 (2003).

${ }^{16}$ H. Seo, S. Aihara, T. Watabe, H. Ohtake, M. Kubota, and N. Egami, Jpn. J. Appl. Phys., Part 2 46, L1240 (2007).

${ }^{17}$ S. Aihara, H. Seo, M. Namba, T. Watabe, H. Ohtake, M. Kubota, N. Egami, T. Hiramatsu, T. Matsuda, M. Furuta, H. Nitta, and T. Hirao, IEEE Trans. Electron Devices 56, 2570 (2009).

${ }^{18}$ S. R. Forrest, MRS Bull. 30, 28 (2005).

${ }^{19}$ J. Nelson, J. J. Kwiatkowski, J. Kirkpatrick, and J. M. Frost, Acc. Chem. Res. 42, 1768 (2009).

${ }^{20}$ L. Chen, Y. Tang, X. Fan, C. Zhang, Z. Chu, D. Wang, and D. Zou, Org. Electron. 10, 724 (2009).

${ }^{21}$ M. Ichikawa, E. Suto, H.-G. Jeon, and Y. Taniguchi, Org. Electron. 11, 700 (2010).

${ }^{22}$ M. Ichikawa, H. Yanagi, Y. Shimizu, S. Hotta, N. Suganuma, T. Koyama, and Y. Taniguchi, Adv. Mater. (Weinheim, Ger.) 14, 1272 (2002).

${ }^{23}$ H. Yanagi, Y. Araki, T. Ohara, S. Hotta, M. Ichikawa, and Y. Taniguchi, Adv. Funct. Mater. 13, 767 (2003).

${ }^{24}$ K. Nakamura, M. Ichikawa, R. Fushiki, T. Kamikawa, M. Inoue, T. Koyama, and Y. Taniguchi, Jpn. J. Appl. Phys., Part 2 43, L100 (2004).

${ }^{25}$ S. Hotta, T. Katagiri, T. Yamao, K. Shimizu, H. Yanagi, M. Ichikawa, and Y. Taniguchi, Int. J. Polym. Mater. 57, 515 (2008).

${ }^{26}$ J. Xue, B. P. Rand, S. Uchida, and S. R. Forrest, Adv. Mater. (Weinheim, Ger.) 17, 66 (2005).

${ }^{27}$ J. K. Bowmaker and H. J. Dartnall, J. Physiol. (London) 298, 501 (1980).

${ }^{28}$ S. M. Sze, Physics of Semiconductor Devices, 2nd ed. (Wiley, New York, 1981). 